Mots. Les langages du politique

Discours d'autorité : des discours sans éclat(s)?

\title{
Couac. Un levier pragmatico-énonciatif dans le discours journalistique
}

Couac. A pragmatico-enonciative lever in the journalistic discourse

Couac. Una palanca pragmático-enunciativa en el discurso periodístico

\section{Annabelle Seoane}

\section{(2) OpenEdition}

Journals

Édition électronique

URL : https://journals.openedition.org/mots/21940

DOI : $10.4000 /$ mots. 21940

ISSN : 1960-6001

Éditeur

ENS Éditions

Édition imprimée

Date de publication : 23 mars 2015

Pagination : 135-152

ISBN : 978-2-84788-698-6

ISSN : 0243-6450

\section{Référence électronique}

Annabelle Seoane, « Couac. Un levier pragmatico-énonciatif dans le discours journalistique », Mots. Les langages du politique [En ligne], 107 | 2015, mis en ligne le 23 mars 2017, consulté le 23 avril 2022. URL : http://journals.openedition.org/mots/21940 ; DOI : https://doi.org/10.4000/mots. 21940 


\section{Couac. Un levier pragmatico-énonciatif dans le discours journalistique}

«Ce gouvernement est à l'ouest, on va de couac en flop. François Hollande avait annoncé qu'il voulait une France apaisée et, finalement, c'est une France déchirée !» (Aujourd'hui en France, 4 février 2014). Tels sont les propos de Brice Hortefeux, ancien ministre UMP, qui ont éveillé notre intérêt. Le terme couac en particulier, par sa sonorité digne d'une onomatopée ou par sa tonalité aussi imagée que familière, conférait à ces propos une incontestable dimension argumentative et une entrée dans le champ médiatique actuel. C'est ce qui a motivé ces quelques réflexions pragmatico-énonciatives sur couac dans le discours journalistique que nous livrons ici.

Le couac est initialement une «fausse note discordante produite par un instrument ${ }^{1}$ (en se teintant d'une coloration politique, son usage dans la presse écrite relève donc de la métaphore; c'est la raison pour laquelle nous l'aborderons sous le jour de son fonctionnement discursif et figural (métaphore, euphémisme, amplification). La reprise de couac à travers les grands titres de presse nous servira de fil conducteur pour appréhender la visée pragmatico-énonciative de cet objet discursif porteur d'enjeux à la fois médiatiques et politiques. Sa reprise sera considérée comme un objet d'analyse, un indice d'une dimension transdiscursive induisant une intentionnalité : réutiliser un terme évaluatif comme couac, au contenu référentiel assez vague, n'est pas anodin pour l'énonciateur car il s'inscrit ainsi dans un interdiscours qui se fonde sur la circularité du terme dans un espace médiatique donné. Terme supposé connu du lecteur, il véhicule un univers de discours qui se construit au fil de ses réemplois et réappropriations. Nous verrons ainsi que cette reprise le rapproche d'un fonctionnement «formulaire», au sens défini par Alice Krieg-Planque (2009, 2012). Si une formule est « un ensemble de formulations qui, du fait de leurs emplois à un moment donné et dans un espace public donné, cristallisent des enjeux politiques et sociaux que ces expressions contribuent dans le temps à construire» (Krieg-Planque, 2009, p.7), nous verrons que le terme couac

1. TLFI, http://atilf.atilf.fr/.

Clesthia-Cediscor, «Sens et discours», Paris 3 Sorbonne nouvelle annabelleseoane@yahoo.fr 
répond à ces caractéristiques malgré son flou référentiel. En effet, d'une part, il cristallise des enjeux discursifs tels que la construction d'une représentation du tiers référentiel, l'action gouvernementale essentiellement - il tend par là à une forme de stéréotypisation de l'attitude du gouvernement -, la construction d'une représentation discursive du locuteur-journaliste, l'ethos, ainsi que des enjeux médiatiques et politiques. Et d'autre part, parce qu'il est au cœur d'un dispositif polémique, il s'ancre dans la situation d'énonciation et dans l'actualité du moment. II montre alors une capacité d'ouverture à d'autres discours (dialogisme interdiscursif, Bres, Nowakowska, 2005) et à des inférences du lecteur qui devient coénonciateur (dialogisme interlocutif). Ces spécificités lui permettent d'entrer dans le débat public. Son flou référentiel et sa circulation dans le champ médiatique lui donnent alors une valeur de levier pragmatico-énonciatif.

Il ne s’agit pas ici de procéder à une analyse lexicale mais de montrer que, en le considérant comme un observable spécifique de phénomènes de construction du sens en discours, couac acquiert une dimension stratégique de levier pragmatico-énonciatif. Chaque emploi devient une réactivation d'un paradigme préexistant qui fait entrer l'énonciateur dans l'arène du discours public, avec ses rapports de pouvoir et d'opinion. Nous aborderons ces questions à travers un plan tripartite dans lequel nous mettrons d'abord en relief son fonctionnement en discours, dans une perspective intra- et interdiscursive, en détaillant ses principales valeurs référentielles et pragmatiques. Nous considérerons ensuite le terme couac comme outil de construction de représentations (par le jeu des chaînes anaphoriques, des tournures figées ou défigées et des discours rapportés) et enfin comme vecteur d'enjeux médiatiques, à travers l'émergence d'un ethos journalistique et le déclenchement d'une mémoire interdiscursive.

\section{Valeurs référentielles et pragmatiques}

En nous fondant sur la base de données Europresse, nous avons constitué un corpus de six cents articles, publiés entre mai 2012 et mai 2014 par la presse généraliste française ${ }^{2}$. L'intervalle considéré correspond aux deux premières années de la présidence de François Hollande et, jusqu'en mars 2014, au gouvernement dirigé par le Premier ministre Jean-Marc Ayrault, qui présente sa démission à la suite de la défaite de la gauche aux élections municipales de mars 2014, puis aux débuts du gouvernement de Manuel Valls. Cet ensemble d'articles permet d'esquisser un bilan de ces deux années de présidence dans

2. Nous avons limité notre réflexion à la presse quotidienne nationale pour des raisons de malléabilité du corpus : Aujourd'hui en France, Le Figaro, Libération, Marianne, Le Monde, Le Point, Valeurs actuelles. Le mode de recueil numérisé adopté ici ne permet malheureusement pas de prendre en compte le statut générique et la place de l'article dans l'économie générale du journal. 
la sphère médiatique de la presse « papier » traditionnelle, à travers l'utilisation itérative du lexème couac par les journalistes. Nous avons observé un lien entre son utilisation et la construction progressive d'une représentation partagée, généralisée presque, de l'action gouvernementale. Pour étayer cet angle d'analyse, nous avons associé couac au mot clef gouvernement. Le nombre d'occurrences de couac + gouvernement dans la presse depuis mai 1981 (élection de François Mitterrand) montre une nette augmentation au fil des années, particulièrement sous la présidence de Nicolas Sarkozy et surtout dans la période qui nous intéresse ici. La moyenne annuelle de ces occurrences en tant qu'indicateur de la fréquence du terme révèle une installation de plus en plus prégnante. Le tableau ci-dessous synthétise ces données dans la presse quotidienne nationale $(\mathrm{PQN})$ et régionale $(\mathrm{PQR})$ :

Tableau 1. Nombre d'occurrences du lexème couac

\begin{tabular}{lrrrc}
\hline & PQN & PQR & Total & Moyenne annuelle \\
\hline mai 2012 - mai 2014 - F. Hollande & 276 & 617 & 893 & 446,5 \\
\hline mai 2007 - mai 2012 - N. Sarkozy & 464 & 507 & 971 & 194,1 \\
\hline mai 1995 - mai 2007 - J. Chirac & 478 & 286 & 764 & 63,7 \\
\hline mai 1981 - mai 1995 - F. Mitterrand & 45 & 3 & 48 & 3,4 \\
\hline
\end{tabular}

Tâchons à présent de tirer parti de cette installation du terme dans le discours médiatique. L'analyse du fonctionnement en discours du terme couac révèle deux axes d'études possibles, qui correspondent aux objectifs pragmatiques de l'énonciateur : d'une part, marquer les esprits et les mémoires en opérant comme procédé repérable formellement autant que comme dispositif formulaire et, d'autre part, marquer son point de vue à travers une énonciation axiologisée et un système de reformulations.

\section{Marquer les esprits et les mémoires}

Selon le moment politique dans lequel il s'inscrit, ce lexème apparaît volontiers à une place cruciale de l'article dans un tiers des articles : titre, chapeau, tout premiers ou derniers énoncés de l'article. Cette position lui attribue une dimension de concentration du sens cataphorique ou bien anaphorique :

\footnotetext{
Ayrault, autopsie d'un couac. (Aujourd'hui en France, 31 octobre 2012)

Couac sur les nouveaux livrets de famille. (Le Figaro, 30 mai 2013)

La loi famille reniée, un énième couac. (Libération, 4 février 2014)

Un gazouillis qui fait couac. (Le Monde, 14 mars 2014)
}

Le reste de l'article vient justifier ce choix lexical, sous la forme de séquences narratives et/ou explicatives. Les titres représentent des « lieux de rappels privilégiés, soit qu'ils fonctionnent en interaction avec des titres antérieurs, soit 
qu'ils usent exemplairement des potentialités que fournit la langue» (Moirand, 2007, p.139). Cette position stratégique permet au couac d'être mis en exergue et de fonctionner dans une forme d'immédiateté. En clôture d'article, il joue également sur la mémoire de l'allocutaire en intervenant comme le mot conclusif qui vient concentrer tout le dispositif info-argumentatif du discours.

En outre, la mise en valeur par des guillemets constitue un autre moyen stylistique et visuel de mettre l'accent sur couac: «Beaucoup plus qu'un énième “couac", l'affaire Léonarda atteste de la confusion qui règne dans la majorité où le PS se révèle incapable d'afficher le minimum de la solidarité gouvernementale » (Libération, 17 octobre 2013). Les guillemets témoignent ici d'une non-adéquation du dire et de son énonciateur, d'une mise à distance de celuici par rapport à ce dit. Ce dit est signalé typographiquement comme appartenant à un autre niveau de lecture, au niveau de l'interdiscours, aussi bien qu'à un registre de langue ressenti comme plus familier. Dans la citation suivante, l'épithète "fameux» place la compréhension de couac sans ambiguïté sur un mode interdiscursif : «Autre cas d'école, si l'on ose dire, le fameux “couac” de Vincent Peillon sur le cannabis : les médias ont unanimement cloué au pilori le ministre de l’Éducation» (Marianne, 31 octobre 2012).

On observe du reste souvent les mêmes combinaisons avec le lexème couac, ce qui contribue à constituer une forme de "mémoire des dires » (Moirand, 2007).

Ainsi, d'une part, si son référent peut semblervariable, le terme est lié souvent aux mêmes ministres : Jean-Marc Ayrault, Christiane Taubira, Vincent Peillon, Arnaud Montebourg, Jérôme Cahuzac, Ségolène Royal, jusqu’à faire partie intégrante de leur désignation : "Ségolène Royal : Madame couac » Valeurs actuelles, 17 avril 2014). Il cristallise autour de ces personnalités une aura de non-professionnalisme, et ces associations récurrentes finissent par ériger ces ministres en figures de proue d'une politique gouvernementale jugée défaillante par la presse.

D'autre part, couac apparaissant rarement seul, ce sont les mêmes adjectifs épithètes qui lui sont régulièrement associés : «nouveau couac » (près de la moitié des occurrences), "un autre couac», "premier couac», "tout dernier couac», qui font explicitement le lien avec d'autres événements antérieurs du même acabit. Ces associations reprises d'un article à l'autre, aussi abondantes que marquées par une temporalité médiatico-politique, font émerger une valeur mémorielle inter- voire transdiscursive, de ce lexème couac. Elles se rapprochent de ce que Moirand évoque comme des «mots qui ont une mémoire » (2007, p. 51) et la récurrence de ces combinaisons fonctionne comme des «rappels mémoriels» (Id., p. 59) avec l'allocutaire. Chaque occurrence de couac devient alors le maillon d'une chaîne qui se construit au fil des articles.

Ce mot se charge donc d'une valeur spécifique, sédimentée, construite sur la mémoire de l'allocutaire et volontiers liée à la vision désabusée de la 
sphère médiatique sur des lignes politiques gouvernementales jugées peu cohérentes. Couac s'inscrit dans un contexte discursif polémique, par le jeu de « rappels mémoriels » qui l'érigent, en définitive, en savoir partagé. Dire couac, c'est entrer dans le débat public, marquer son point de vue face à un tiers référentiel, la gouvernance de J.-M. Ayrault dans notre corpus. Il opère ainsi une cristallisation de positions antagonistes qui affleurent dans le discours : positions critiques face à la communication des membres du gouvernement en tant qu'équipe et postures dubitatives (voire sarcastiques) quant à l'action gouvernementale dépeinte comme défaillante.

\section{Marquerson point de vue}

Ces positions antagonistes apparaissent de différentes façons : le réseau sémantique autour du lexème couac au sein d'une énonciation axiologisée et/ ou distanciée, le système parfois complexe de reformulations à l'intérieur d'un même article et enfin, l'énonciation distanciée mise en œuvre par le recours à des discours explicitement rapportés.

Les adjectifs axiologiques marquent un jugement de valeur de la source locutive, le journaliste : «ce fâcheux couac » (Le Monde, 21 mai 2014), « regrettable couac» (ibid., 28 février 2014), " un couac majeur (ibid., 7 décembre 2013), « un gros couac » (Libération, 10 août 2013, Aujourd'hui en France, 17 mai 2012), « un impensable couac juridique » (Aujourd'hui en France, 8 août 2013), «un couac inquiétant» (Le Monde, 25 juillet 2013), « un couac des plus inquiétants» (L'Humanité, 6 août 2013). Le socle prédicatif induit également une axiologie : «empêtré dans un couac » (L'Humanité, 26 février 2014), « a viré au couac » et «être pris en défaut du moindre couac» (Le Figaro, 4 avril 2014). Mais c'est dans l'opération de reformulation que résonne le mieux la tonalité négative de couac. En effet, les journalistes ont recours à des reformulations, tantôt explicatives, tantôt interprétatives, qui s'inscrivent dans ce qui s'apparente à une sorte de typologie des actions du gouvernement. Les différents « couacs» deviennent ponctuellement des «bévues» (Le Figaro, 25 et 26 octobre 2012), des «bourdes» (ibid., 25 octobre 2012), un «faux pas» (ibid., 31 octobre 2012), des «ratés» (ibid., 25 octobre 2012, Aujourd'hui en France, 5 février 2014), des «gaffes» (Libération, 25 octobre 2012), une "invraisemblable boulette» (ibid., 17 octobre 2012), « un gros cafouillage» (ibid., 13 septembre 2013) ou «un cafouillage symbolique» (Le Figaro, 16 mars 2013).

On trouve des désignations qualifiantes, à valeur dépréciative plus générale données comme des équivalences à couac, comme «incident» ou «dysfonctionnement» 3 :

3. Les italiques dans les citations sont de nous et visent à faire ressortir certains éléments qui font l'objet d'un commentaire. 
Le premier incident a eu lieu à Marseille lors des journées d'été des écologistes où Martin a annoncé une contribution énergie climat faisant bondir certains socialistes qui dénonçaient dans le même temps le «ras-le-bol fiscal» [...]. Le deuxième couac a eu lieu mercredi dernier lors d'une conférence de presse au cours de laquelle il a laissé entendre qu'il n'y aurait pas d'augmentation du diesel, provoquant aussitôt une crise gouvernementale avec les écologistes. (Aujourd'hui en France, 14 septembre 2013)

Un couac au Sénat met le gouvernement en porte-à-faux. La gauche s'est retrouvée minoritaire hier au moment du vote sur le durcissement des droits de succession voulu par le gouvernement. Dysfonctionnement de taille hier matin au Sénat. (La Croix, 27 juillet 2012)

D’autres reformulations s'inscrivent dans la controverse médiatique : «un beau pataquès », «imbroglio», «un bug»:

Le couac de Jean-Pierre Jouyet sur «les canards boiteux». Le futur président de la Banque publique d'investissement a déclenché un beau pataquès en disant, vendredi sur Europe 1, qu'elle n'avait pas vocation à "aider les canards boiteux», comme le site de Florange. (Libération, 20 octobre 2012)

Et le premier couac oppose... Bercy au Quai d'Orsay. À peine formé, le gouvernement Valls est le théâtre d'un premier imbroglio, opposant Fabius et Montebourg pour la tutelle du commerce extérieur. (Le Monde, 4 avril 2014)

Loi Duflot censurée : Chronique d'un «bug» annoncé. Le gouvernement se serait bien passé de ce nouveau couac sur une promesse phare du candidat Hollande. Cette fois, c'est par excès de zèle qu'il a péché. (Le Figaro, 25 octobre 2012)

Lorsque la difficulté prend une plus grande dimension médiatique, le terme affaire apparaît comme pendant lexical. Dans la citation suivante, il réfère aux écoutes téléphoniques dans une procédure juridique à l'encontre de Sarkozy, désignation communément reprise par l'ensemble de la presse, mais ici le journaliste utilise également affaire pour évoquer la mauvaise communication entre le président et les ministres informés de «l'affaire des écoutes de Sarkozy », ce qui constitue un raté, un couac. La combinaison des deux référents dans la tournure anaphorique «cette affaire dans l'affaire » donne une connotation dramatisante :

La gouvernance Hollande en question. Ce nouveau couac souligne les faiblesses du dispositif et conforte l'hypothèse d'un remaniement. [...] Cette affaire dans l'affaire est d'abord un énorme raté de communication, qui jette une lumière crue sur le manque de coordination au sommet de l'État. (Le Figaro, 13 mars 2014)

Par rapport à affaire, couac apporte une légèreté par sa sonorité d'onomatopée et une dimension itérative puisqu'il est associé à l'adjectif nouveau dans ses deux occurrences. L'association des deux termes renforce le point de vue à charge du quotidien marqué à droite par une touche de sensationnalisme.

Plus généralement, ces reformulations renvoient aussi bien à la nature du dysfonctionnement qu'à ses conséquences et leur accumulation prime dans 
une lecture globale des articles de presse afférant à l'action du gouvernement. Ces désignations se rapprochent de procédés métonymiques (contiguïté de la cause pour l'effet) : «le flou» (Aujourd'hui en France, 4 avril 2014), «la confusion» (Libération, 17 octobre 2013), «ce rétropédalage » (Aujourd'hui en France, 7 septembre 2012, Le Figaro, 16 octobre 2012). On observe également d'autres tournures prédicatives comme "la machine s'emballe»:

Mariage gay : Taubira part avec un temps d'avance. Hier, la garde des Sceaux a dévoilé le projet d'union pour tous. Sans la ministre de la Famille et en volant la vedette à un texte d'EE-LV. Est-ce un coup, un couac, ou tout simplement un paquet cadeau? Alors que le calendrier du projet de loi du gouvernement sur le mariage pour tous était enfin fixé et rendez-vous pris en Conseil des ministres le 31 octobre, la machine s'emballe. (Libération, 12 septembre 2012)

Ces reformulations relèvent d'abord d'un jugement sur la communication du gouvernement, mais également d'un commentaire sur l'action du gouvernement. Les deux entrant dans un rapport métonymique, comme le soulignent parfois certains journalistes. C'est le cas de cet article du Figaro, qui s'interroge sur la portée des «couacs» du gouvernement:

Mensonge caractérisé ou maladresse incontrôlée : la bourde de Christiane Taubira ressemble au couac de trop, au couac final d'un gouvernement qui vient d'apporter l'argument ultime de l'urgence d'un remaniement. Ces versions contradictoires, incomplètes, successives ou fausses sur la connaissance au sommet de l'État des écoutes de Nicolas Sarkozy ont des airs de bouquet final ravivant tous les travers et les mauvais démons de l'équipe Ayrault depuis deux ans. [...] Il y a ensuite cet abonnement aux couacs, onomatopée devenue le mot générique de la gouvernance socialiste. Un premier ministre se rendant au 20 heures de France 2 pour contredire ce qu'a dit sa garde des Sceaux la veille dans le 20 heures de TF1. Cette contradiction a rendu inaudible l'argumentaire gouvernemental. C'est l'une des lois de la communication politique : en cas de divergence au sommet, l'opinion ne s'attache plus à la pertinence ou au bien-fondé d'un discours, pour ne plus s'interroger que sur les motifs des contradictions. Avec la certitude que le flou dans l'expression masque la malveillance des intentions. (Le Figaro, 13 mars 2014)

Ces reformulations métonymiques des «couacs» deviennent un indicateur de l'impuissance politique : la non-compréhension des enjeux du dire dans la sphère publique ne fait que dévoiler les difficultés à mener une action collective efficace. La métonymie s'entend également à ce niveau.

Ces reformulations introduisent ou closent une séquence narrative de longueurvariable qui vient justifier l'emploi de couac. Cette séquence raconte l'enchaînement narratif que le journaliste désigne anaphoriquement ou cataphoriquement par couac et ancre clairement le terme, du point de vue référentiel, dans la communication politique, jugée point faible du gouvernement : "Un couac majeur dans la communication de l'Élysée?» (Le Monde, 29 novembre 2013), «Controverse du Net. Plus qu'un simple couac de communication, le 
geste de la "première dame” a immédiatement dégénéré en "Trierweilergate” " (Le Monde, 16 juin 2012). Ramasser le contenu de la séquence narrative en un seul terme permet au journaliste de développer un positionnement axiologique tout en marquant les esprits. La valeur d'onomatopée de couac sert a fortiori cette visée pragmatique.

Les journalistes tirent d'ailleurs également parti du référent initial du terme pour décliner la métaphore musicale : «fausses notes de ce gouvernement» (Marianne, 27 octobre 2012), des «bafouillements monocordes, sa communication indigente» (Libération, 7 octobre 2013). Signe que ces désignations dépassent le simple clivage idéologique droite/gauche, on retrouve la même métaphore de la «cacophonie» dans des journaux comme Le Figaro (4 mai 2013) ou L'Humanité (3 octobre 2013). Ce dernier utilise d'ailleurs un complément de nom pour axiologiser encore la qualification, "une cacophonie de force 10 sur le couac de Richter»: la double métaphore "sur le couac de Richter » condense celle de l'échelle de mesure de la magnitude du séisme et celle du couac, elle ajoute une gradation à la tonalité moqueuse de ce discours.

Ces désignations qualifiantes permettent de prendre position selon la ligne éditoriale du journal et construisent peu à peu, en se fondant sur leur accumulation et leur convergence, une représentation médiatique subjectivante de la communication et de l'action du gouvernement. Elles permettent d'appréhender ce que le journaliste entend par couac mais également d'en préciser les conséquences communicationnelles et politiques (manque de clarté, de cohérence au sein du gouvernement). Ses reformulations vont de pair avec le déploiement d'une doxa - nous y reviendrons. Étudions à présent comment le recours à couac permet de construire une représentation (trans)discursive du gouvernement, mais également du locuteur.

\section{Un outil de construction de représentations}

Ces reformulations qualifiantes récurrentes construisent une représentation axiologisée de ce tiers qu'est, peu ou prou, le gouvernement Ayrault dans notre corpus. Cette construction dans et par ces discours s'opère par le maillage discursif de chaînes anaphoriques, par le jeu de tournures figées, défigées, et enfin par le biais de discours rapportés. Nous verrons que ces discours sont traversés par d'autres discours, s'inscrivant ainsi dans une logique de dialogisme interdiscursif utilisée à des fins pragmatiques.

\section{Par des chaînes anaphoriques}

Si l'on observe ces reformulations d'un point de vue macrodiscursif, on constate que certains articles utilisent un système de chaînes anaphoriques 
assez dense pour construire une représentation, largement à charge, de l'action gouvernementale, comme dans l'article ci-dessous issu du Figaro, déjà cité supra (13 mars 2014), marqué idéologiquement à droite :

Une crédibilité en ruine au sommet de l'État.

Mensonge caractérisé ou maladresse incontrôlée : la bourde de Christiane Taubira ressemble au couac de trop, au couac final d'un gouvernement qui vient d'apporter l'argument ultime de l'urgence d'un remaniement. Ces versions contradictoires, incomplètes, successives ou fausses sur la connaissance au sommet de l'État des écoutes de Nicolas Sarkozy ont des airs de bouquet final ravivant tous les travers et les mauvais démons de l'équipe Ayrault depuis deux ans. À commencer par ce soupçon d'amateurisme que le gouvernement n'aura jamais réussi à conjurer. [...] II y a ensuite cet abonnement aux couacs, onomatopée devenue le mot générique de la gouvernance socialiste. Un premier ministre se rendant au 20 heures de France 2 pour contredire ce qu'a dit sa garde des Sceaux la veille dans le 20 heures de TF1. Cette contradiction a rendu inaudible l'argumentaire gouvernemental. [...] Avec la certitude que le flou dans l'expression masque la malveillance des intentions. À tort ou à raison, ce mal-là est fait. [...] Il ne faut pas s'étonner non plus si cet embrouillamini sur les écoutes reçoit un démenti de plus de la part du procureur général de Paris, François Falletti, que la garde des Sceaux avait tenté d'écarter de son poste. Du coup, c'est l'écart entre les grandes proclamations sur l'indépendance de la justice et la réalité des méthodes utilisées qui apparaît aussi au grand jour. Du fait de ce mensonge que Jean-Marc Ayrault a été contraint de reconnaître, c'est donc toute la crédibilité de la parole gouvernementale qui est entachée. Et sans lien sur le fond avec les autres fiascos ayant rythmé l'action de l'exécutif, celui-ci réveille le souvenir de la parole de l'État ridiculisée dans l'affaire Leonarda, de la promesse présidentielle foulée aux pieds sur le chômage. À moins de dix jours du premier tour des municipales, la gauche prend en pleine face cette démonstration d'amateurisme.

De nouveau, il y a ici à la fois des reformulations qualifiantes qui détaillent ce que le journaliste désigne d' "abonnement aux couacs, onomatopée devenue le mot générique de la gouvernance socialiste». La double métaphore du couac associé à "abonnement» qui en souligne la récurrence, vient étayer les métaphores de «la crédibilité en ruine», annoncée en titre et de la crédibilité «entachée» de la parole gouvernementale. La chaîne d'anaphores exophoriques soudée à coups de démonstratifs aboutit au dernier énoncé de l'article. Se construit, anaphore après anaphore, une représentation très imagée de cet «amateurisme».

\section{Parle jeu de tournures figées ou défigées}

Si l'accroissement de la fréquence d'un terme témoigne de son installation dans les discours, il présente une forme de stabilisation qui lui donne une dynamique formulaire, nous semble-t-il. En effet, «une formule présente à la fois un caractère figé, une existence en discours, une valeur en tant que référent 
social et une dimension polémique » (Krieg-Planque, 2012, p. 111). Il devient un terme clef dans l'argumentation développée à l'intérieur de certains discours car, on l'a vu, le réseau de désignations qu'il instancie acquiert une valeur pragmatique forte de construction, dans notre corpus, d'une représentation négative de l'action gouvernementale. C'est bien la récurrence convergente des «couacs» utilisés qui contribue à fonder cette représentation de non-cohérence et de non-cohésion : "amateurs » (Le Figaro, 13 avril 2014), «ce gouvernement de bric et de broc [...] un tel mélange d’incompétence abyssale et de prétention stratosphérique, qui a banalisé le mot "couac" comme sa forme habituelle de "communication" " (Valeurs actuelles, 10 avril 2014), «les pieds nickelés» (Le Point, 24 octobre 2013).

Sans être précisément une formule, le lexème couac tend néanmoins à endosser un fonctionnement formulaire par l'association de plus en plus récurrente avec la politique socialiste du gouvernement que sa circulation dans le discours de presse suscite. Les journalistes ont recours à des formulations figées pour fixer cette association entre les couacs à répétition et un gouvernement décrit comme peu professionnel et utilisent, principalement dans les titres, des défigements issus d'objets de discours populaires, comme un titre d'album de la bande dessinée de Tintin «Couacs en stock» (Valeurs actuelles, 21 mars 2013), d'expressions populaires ou "têtes à couac», "un couac d'avance », de chansons " pour un couac avec toi » (Le Figaro, 25 octobre 2012). Ces jeux de mots donnent une tonalité moqueuse à leur discours et tablent sur une connivence avec le lecteur, ce qui a, évidemment, une incidence sur son ethos. Ils installent un rapport critique au pouvoir politique et un rapport structurant à l'opinion publique. Ces rapports transparaissent tout particulièrement dans le jeu des discours rapportés.

\section{Par le biais des discours rapportés}

Le recours fréquent à des discours rapportés marque la volonté d'ouverture à d'autres discours exogènes, tenus par des politiques, membres du gouvernement, du PS ou de l'opposition. Couac prend alors véritablement une dimension de pivot énonciativo-argumentatif car d'une part, il sert de mot qui condense le commentaire distancié de la part de l'énonciateur journaliste, face à des propos cités, ressentis comme euphémisants, et en ce sens, il est souvent rejeté par la classe politique. D'autre part, il sert de fixateur de cet interdiscours journalistique et certains politiques se l'approprient (pour restaurer leur image, se placer dans l'air du temps, montrer une connivence avec la presse et donc un discours sans langue de bois ou sans faux-semblants...). En effet, couac constitue par anaphore ou cataphore une version condensée d'une séquence narrative à portée explicative d'un événement politique précis, donné comme mal géré par le gouvernement. Lorsque des propos rapportés interviennent, couac se 
place du côté du commentaire journalistique, revêtant, dans un premier temps du moins, une aura d'objectivité face à des reformulations qui apparaissent comme des procédés d'euphémisation par les politiques.

Sous la forme de discours direct ou indirect, les propos rapportés traduisent une subjectivité de la part de l'énonciateur4. Ces propos sont introduits par des verbes tels que gronde, s'agace, grincer, regrette, exaspérer, persifle, râle, condamne par opposition à des verbes comme argumente, relativise, temporise, assure... Ces verbes du dire traduisent la volonté du journaliste de mettre en mots un point de vue, un positionnement énonciatif autant que politique, de l'énonciateur détracteur du gouvernement ou, au contraire, de celui qui se pose en défenseur, voire garant de la ligne politique du gouvernement. Par exemple, là où l'énonciateur-journaliste mentionne clairement un «couac », l'ancienne porte-parole du gouvernement, Najat Vallaud-Belkacem, parle de «maladresse» (L'Humanité, 3 octobre 2013), un dirigeant du groupe PS explique un simple «problème de timing» (Le Figaro, 25 octobre 2012), l'élu PS Julien Dray d' « un problème de cohérence et d'explication » (L'Humanité, 3 octobre 2013), l'ex-ministre Delphine Batho explique que «C'est vrai qu'on ne comprend plus grand-chose » (Le Figaro, 17 septembre 2013), et ce, même si l'utilisation de couac peut, plus rarement, venir rééquilibrer les propos amplifiants, dramatisants, d'une opposition : «Mais, à droite, on n'a bien sûr pas manqué d'exploiter ce nouveau couac gouvernemental. [...] Copé dénonce « un spectacle lamentable [...] Le Premier ministre est terriblement affaibli, il n'a plus de crédibilité, a-t-il insisté» (Le Parisien, 25 février 2014).

Dans certains articles, le mot couac apparaît comme un pivot argumentatif central dans le métacommentaire journalistique d'un incident politique. $A$ contrario, les membres du gouvernement tâchent, eux, de minimiser l'épisode, ce qui donne lieu au déploiement d'une rhétorique centrée sur l'euphémisation ou «bémolisation du discours» (Jaubert, 2008, p. 112) :

Du côté des écologistes, on s'attache donc désormais à dédramatiser ce couac. (La Croix, 19 septembre 2012)

"ll y a eu un peu d'empressement, de précipitation », euphémise-t-on au ministère de la Famille, en toute diplomatie. «Ce texte est un habillage juridique de l'engagement du Président destiné à servir de socle commun à la discussion », aplanissaiton Place Vendôme hier, en arguant que, «quand on modifie les dispositions essentielles du code civil, on a forcément un projet élaboréà la chancellerie. " (Libération, 12 septembre 2012)

4. Nous nous plaçons dans la continuité de Rabatel (2010, p. 370), entre autres, pour la distinction locuteur/énonciateur : « Le locuteur est l'instance première qui produit matériellement les énoncés [...] L'énonciateur est l'instance qui se positionne par rapport aux objets du discours auxquels il réfère, et, ce faisant, qui les prend en charge. La notion d'énonciateur correspond à une position (énonciative) qu'adopte le locuteur, dans son discours, pour envisager les faits, les notions, sous tel ou tel point de vue.» 
Après avoir rétropédalé, le cabinet de Filippetti tente aujourd'hui de minimiser le couac. (Aujourd'hui en France, 6 juillet 2012)

En contrepoint de ces discours à charge, émerge un dispositif d'objectivation des dires et des faits dans lequel couac a un rôle phare. Il tend dès lors à devenir emblématique du discours journalistique et est, à ce titre, repris par la classe politique, dans une posture soit de réfutation, soit de réappropriation.

L'exemple suivant montre l'attribution explicite du couac au discours journalistique par un politique et sa tentative de minimisation de la portée du terme :

Le bras de fer entre Valls et Taubira sur la réforme pénitentiaire à l'été 2013, ensuite : «Le premier ministre a minimisé l'affaire. Pour lui, c'était un débat, pas un couac.» (Le Figaro, 22 février 2014)

Un différend politique, leur disait-elle [N. Vallaud-Belkacem] ce n'est pas un couac. (Libération, 2 novembre 2012)

Ces propos entrent dans le cadre d'une stratégie de réfutation et de dédramatisation du terme, considéré comme exogène car propre au point de vue des journalistes. En le réfutant, en le recatégorisant par des tournures euphémisantes, le politique s'inscrit en faux et marque sa solidarité avec le gouvernement en même temps qu'il discrédite une presse dépeinte en filigrane comme sensationnaliste.

Àl'inverse, certains politiques n'hésitent pas à réutiliser couac faisant allusion à des événements antérieurs :

Un autre député s'étonne de la rapidité avec laquelle l'exécutif a changé de braquet. "Oui, c'est un couac, un de plus, confie-t-il. Et je suis surpris de la brutalité avec laquelle tout cela s'est fait.» (Le Figaro, 5 février 2014)

Quant à Bertrand Delanoë, statue du commandeur, il est à sa place dans l'histoire parisienne : "Il n'y aura pas de rupture, mais je vais passer à un changement d'échelle quand je serai maire», annonce Hidalgo. Ses collaborateurs croisent les doigts : "Jusqu'ici, zéro couac, un sans-faute!» se réjouit le sénateur PS David Assouline. (Libération, 9 décembre 2013).

Il nous semble que dans ces occurrences, le seul intérêt à avoir recours à couac est de convoquer un interdiscours dans/par lequel l'énonciateur sait que s'est construite une représentation du microcosme politique, et de faire de cette manière appel à la mémoire discursive du lecteur pour réorienter cette représentation ou au contraire la conforter. L'infiltration de l'interdiscours journalistique dans ces discours rapportés de personnages politiques contribue à la circulation du mot autant qu'à se reconstruire un ethos : de courage politique et de transparence avec la presse, voire de connivence avec les Français :

«Ce n'est pas un couac, c'est une connerie, une erreur de communication», reconnaît sans fard Lebranchu. (Le Figaro, 19 juin 2013) 
«Quand on ne répond pas, on manque de courage politique, on louvoie. Et quand on répond, c'est de l'ego ou un couac ", s'emporte [C. Taubira], tout en assurant ses arrières dans un déploiement de séduction tous azimuts. (Libération, 19 mars 2013)

Parce qu'il opère comme un levier pragmatico-énonciatif, reformulé, réfuté ou réapproprié, le mot couac entre de plain-pied dans le débat public; il agit comme un pivot dialogique, faisant basculer l'énonciation d'un énonciateur à l'autre, du discours du journaliste à celui du politique ou inversement, et du discours à l'interdiscours. Sa circulation lui instancie une valeur pragmatique d'autant plus forte qu'il est (re)connu par le lecteur et son emploi est le lieu et l'enjeu d'une connivence. À travers cette énonciation distanciée par le moyen de discours rapportés, il construit dialogiquement au fil du discours un ethos spécifique que vient cristalliser le lexème couac.

En cela, l'utilisation de couac laisse entrevoir un ancrage discursif qui se fonde sur sa relation à une temporalité et à des acteurs sociaux, portée par certains locuteurs ou des événements qui la favorisent. Ces événements, notre corpus le montre, tiennent surtout d'erreurs dans la communication collective gouvernementale, plus que de l'efficience ou de la pertinence des mesures prises. En faisant circuler le mot couac, journalistes et politiques contribuent à forger la représentation réductrice d'une équipe gouvernementale maladroite et discordante dont finalement le point d'achoppement principal réside dans sa non-maîtrise des enjeux de la communication publique collective. Le discours de la presse, par la reprise d'un mot comme couac d'un locuteur à l'autre, façonne peu à peu l'opinion publique en même temps qu'il la conditionne à subir incidemment sa propre influence.

\section{Un vecteur d'enjeux médiatiques}

\section{L'émergence d'un ethos journalistique}

Nous venons d'aborder la question de couac au cœur d'une énonciation distanciée, où la circulation des dires en tant que mise en œuvre d'un dialogisme interdiscursif (Bres, Nowakowska, 2005) joue un rôle déterminant. En effet, ce dispositif devient propice à l'émergence d'un ethos journalistique par la mise en scène de dires exogènes qui traversent son discours : des propos attribués explicitement ou non tenus par d'autres, en particulier des politiques, on l'a vu, mais également des reprises de termes propres à l'interdiscours journalistique. En tant que source locutive, le journaliste porte une attention particulière à éclairer, orienter la réception que son lecteur peut avoir de ce lexème couac. Il construit un ethos distancié, en se montrant capable de retravailler les tentatives d'euphémisation ou de dramatisation du discours par les énonciateurs qui s'avèrent parties prenantes des différentes lignes politiques. Il fournit des 
reformulations à ces «couacs» et permet d'agir en pivot énonciatif pour dessiner au fil des articles et des reprises du terme une représentation donnée pour objective (ou objectivante, du moins) du gouvernement.

Afin de mieux répondre à cette attente sous-jacente du lecteur, s'ajoute à cette tonalité explicitement orientée vers d'autres discours un « soubassement doxique » prégnant (Amossy, 2000, p.36), c'est-à-dire le fondement sur des prémisses présentées comme admises et entérinées par tous et donc emportant aisément l'adhésion, par exemple via le recours à des questions rhétoriques, ou à des modalisateurs comme «nul doute que ", "évidemment», etc.

Le dialogisme interdiscursif se trouve ainsi doublé dans certains articles d'un dialogisme interlocutif mis en scène par le locuteur-journaliste qui "S'adresse à un interlocuteur sur la compréhension-réponse duquel il ne cesse d'anticiper» (Bres, Nowakowska, 2005, p. 139) et qui transparaît par exemple par des interrogations explicites ou des points de suspension : il appartient au lecteur de répondre à la question ou de compléter la fin de l'énoncé avec ce qui est non dit :

Est-ce la façon socialiste de combiner Realpolitik et droits de l'homme? Hélas, deux ans d'expérience nous montrent assez que tout est à craindre... (Valeurs actuelles, 10 avril 2014)

Le locuteur table alors sur la force persuasive des sous-entendus pour amener le lecteur à faire l'autre moitié du chemin et à formuler lui-même cette thèse pour renforcer son adhésion. La perspective dialogique sous-jacente nous permet d'entrevoir les incidences pragmatiques à l'œuvre et la construction d'une connivence qui s'opère dans ces discours. En faisant implicitement valider son dire par le lecteur, la stratégie du journaliste procède d'une validation de soi par le positionnement énonciatif. Il en va de même pour faire déconstruirereconstruire les défigements à l'œuvre dans certains quotidiens (Le Figaro et Libération essentiellement) ; le lecteur doit recontextualiser le dit pour saisir les apports de ces jeux de mots; il entre ainsi dans une dynamique d'adhésion au dire du locuteur. Par le biais de l'allusion, le renvoi à un extérieur discursif participe à des dynamiques de conflictualité dans le discours. «Défiger un énoncé sur le mode plaisant [c'est] établir la connivence par l'activation d'une mémoire» (Krieg-Planque 2012, p. 192-193).

En outre, le dialogisme interlocutif consiste également, pour la voix journalistique, à anticiper les réactions du lecteur (le doute, l'incompréhension...) - ou à les feindre -, quitte à se placer sur une tonalité qui pourrait sembler narquoise ("Il y a une semaine, la presse révélait l'information. Hollande avait nié. Voilà qui ressemble quand même à un couac de communication. » Libération, 29 juin 2012). «Voilà» et " quand même» témoignent de cette entente tacite avec le lecteur.

Sur fond de visée informative, ethos distancié et ethos de connivence 
concourent en filigrane à la construction d'un ethos journalistique spécifique. L'ethos apparaît donc comme une image travaillée qui émerge à travers la convergence d'un faisceau d'articles où le mot couac se révèle déterminant. Notre intérêt pour la manière dont les marquages du discours élaborent un ethos et révèlent ainsi une prise en charge énonciative caractérisée par les constituants de rangs micro-textuel et macro-textuel a révélé un ethos du journaliste dont la vision distanciée tend à trancher avec la représentation d'un gouvernement d'amateurs et un ethos de connivence qui table sur la volonté de faire du lecteur un coénonciateur. Celui-ci participe en bonne intelligence à la compréhension des enjeux médiatico-politiques de ce couac.

\section{Les médias comme déclencheur d'une mémoire interdiscursive}

En reprenant au fil des mois l'association couac et gouvernement, avec les variations dues à la période/à l'événement politique, on observe que les médias «construisent une mémoire interdiscursive» (Moirand 2007, p. 107) qui se forge sur des dires antérieurs, des reformulations, des allusions et, par là même, la compréhension d'un lecteur averti. Cette démarche émane d'une orientation pragmatique, parfois argumentative, à tel point qu'elle s'inscrit dans la ligne éditoriale plus que réellement idéologique, nous semble-t-il, des journaux de notre corpus.

On remarque ainsi que les principaux titres de la presse française concentrent à eux seuls $88 \%$ des articles avec couac et que, par exemple, Le Figaro, ancré idéologiquement à droite, utilise beaucoup de tournures reformulantes, volontiers axiologisées, et propose de cette manière un nombre d'occurrences et un réseau sémantique plus riches que la plupart des autres, comme le montre la concentration de la répartition des principaux titres de la PQN en fonction du nombre d'articles intégrant au moins une fois le terme couac: $34 \%$ pour Le Figaro, $32 \%$ pour Le Monde, $22 \%$ pour Libération.

Ce qui prime dès lors, c'est bien la redondance du terme couac et cette circularité inter-, voire transdiscursive s'érige en «mémoire partagée» (Moirand 2007, p. 63) avec le lecteur dans la sphère médiatique et donc à son entrée en jeu dans le débat social sous-jacent. Il s'agit avant tout de mobiliser l'opinion.

La circularité de couac est révélatrice d'une prise de position que nous pourrions qualifier de collective par les grands médias; peuvent s'ensuivre des débats sur le débat: des commentaires métadiscursifs sur le terme couac en luimême et ses enjeux médiatiques plus globaux. Ces commentaires deviennent le lieu où le journaliste à l'origine du métadiscours s'inscrit dans cet interdiscours et se positionne à son tour dans le débat public. Comme le mentionne A. Krieg-Planque (2012, p. 117) : «La réflexivité langagière, par laquelle les locuteurs prennent les mots pour objets de leur parole, est alors constituée en point d'observation privilégié : en commentant le mot lui-même, les locuteurs 
donnent à voir un aspect de la position qu'ils occupent. » Les deux extraits suivants montrent la prise de position adoptée par deux journalistes. Le premier relève d'une mise à distance de discours ambiant autour de couac en légitimant la bémolisation du couac par N. Vallaud-Belkacem; le second, la manifestation d'une position qui se donne comme critique et originale :

Reconnaissons que Najat Vallaud-Belkacem n'avait pas tort, mercredi, de renvoyer à leurs dictionnaires les porte-micros la chatouillant à propos de ce qu'il est très à la mode de qualifier de "couacs de communication». "Un différend politique, leur disait-elle, ce n'est pas un couac. " Elle a raison. Et si les «différends politiques» constituent aujourd'hui ce que le PS produit de mieux, ce n'est pas forcément une mauvaise nouvelle. (Libération, 2 novembre 2012)

Ils disent tous la même chose... Médias : les moutons de Panurge. [...] Écoutons les radios, regardons les journaux télévisés, lisons les journaux - y compris Marianne : est-il question de l'action du gouvernement qu'invariablement, depuis des semaines, reviennent les mêmes mots, les mêmes expressions, le même champ lexical. «Couacs», «couacs», «couacs »... Comme s'il n'y avait pas d'autre grille de lecture. Un ministre qui contredit un autre ministre : c'est un couac. Vincent Peillon qui ose livrer son sentiment sur la dépénalisation du cannabis : encore un couac. Bercy qui recule, à tort ou à raison, sur telle mesure fiscale : toujours un couac. Questions : dans quel pays tous les ministres sont-ils toujours d'accord entre eux, sinon dans un régime autoritaire? Les fausses notes de ce gouvernement sont-elles vraiment plus fréquentes que dans les précédents? Par définition, les moutons de Panurge du journalisme ne se posent pas ces questions. [...] Une hystérie médiatique chasse l'autre. (Marianne, 31 octobre 2012)

Couac apparaît ici comme la cristallisation de cette «hystérie médiatique». En disqualifiant l'usage du terme, le locuteur se plaît ainsi à sortir de ce qu'il décrit comme un entre-soi jubilatoire des médias. Dans un cadre fortement dialogique, le métadiscours renforce donc la coénonciation du lecteur recherchée par le locuteur-journaliste.

Par un système de renvois interdiscursifs, dire couac fonctionne comme un stimulus qui déclenche une mécanique pragmatique de reconnaissance par le lecteur, puis un travail inférentiel, établissant une connivence inter-journalistique et avec le lecteur.

En conclusion, s'il fait sens ailleurs que dans ces discours, le lexème couac acquiert ici une dimension stratégique de levier pragmatico-énonciatif. Il fait pleinement sens car il se fonde sur une interdiscursivité : les reprises de couac se font écho les unes aux autres et marquent un positionnement énonciatif aussi bien qu'une stratégie pragmatique. À chaque réactivation, d'autant plus si elle est explicite, on entre dans cet interdiscours médiatique et il se crée une relation de connivence, voire un sentiment de «faire communauté » pour certains de ces discours. Ce "faire communauté », pour être plus efficace, doit être fermement ancré dans le moment de réception, tant d'un point de vue discur- 
sif par un embrayage marqué, que d'un point de vue extradiscursif puisqu'il s'imprègne de l'actualité. Cette mise en œuvre se rapproche d'un fonctionnement formulaire puisque «le locuteur manifeste, comme ressort de sa création verbale, un modèle préexistant qui pose celui-ci comme un terme supposé connu des auditeurs-lecteurs» (Krieg-Planque, 2012, p.116). En contribuant à la circulation d'un mot comme couac, le locuteur entre dans l'arène du discours public, avec ses rapports de pouvoir et d'opinion. Comme il oscille entre configuration discursive et instrumentalisation communicationnelle, employer le mot couac avec l'aura qu'il véhicule aujourd'hui dans le discours journalistique ambiant et les valeurs idéologiques qu'on peut lui prêter, permet certes de dessiner les contours d'une représentation polémique de l'action gouvernementale par exemple, mais cela permet surtout, par un stratégique effet de levier, de trouver ou d'affirmer sa place dans la sphère médiatique. N'est-ce pas là, finalement, tout l'enjeu du dire public aujourd'hui ?

\section{Références}

AlÉn Garabato Carmen, 2013, «Bling-bling. Du hip-hop aux dictionnaires, en passant par les médias », Mots. Les langages du politique, nº101.

Amossy Ruth, 2000, L'argumentation dans le discours. Discours politique, littérature d'idées, fiction, Paris, Nathan Université.

BRES Jacques, NowAKowSKA Aleksandra, 2005, «Dis-moi avec qui tu “dialogues”, je te dirai qui tu es... De la pertinence de la notion de dialogisme pour l'analyse du discours », Marges linguistiques, nº 9, p.137-153.

FLoREA Ligia Stela, 2013, «Mise en scène du dit rapporté dans la presse généraliste. L'exemple du quotidien Le Figaro ", Semen, n³ 35, Besançon, Presses universitaires de Franche-Comté, p. 170-187.

JAUBERT Anna, 2008, «Dire et plus ou moins dire. Analyse pragmatique de l'euphémisme et de la litote», Langue française, nº160, p. 105-116.

KRIEg-PlANQUe Alice, 2009, La notion de «formule» en analyse du discours. Cadre théorique et méthodologique, Besançon, Presses Universitaires de Franche-Comté, $144 \mathrm{p}$.

- 2012, Analyser les discours institutionnels, Paris, Armand Colin.

MoIRAND Sophie, 2007, Les discours de la presse quotidienne, Paris, PUF.

RABATEL Alain, 2010, "Retour sur les relations entre locuteurs et énonciateurs. Des voix et des points de vue », Recherches linguistiques, n³1, p. 357-373. 